\title{
Morphological and histochemical changes in muscle biopsies of myasthenia gravis with a typical clinical presentation
}

\begin{abstract}
The morphological and histochemical changes in muscle biopsies from 6 cases with myasthenia gravis (MG) were studied, The patients were misdiagnosed at the initial presentation, however they were all proved later by either histochmical and serological tests or improvement on mestinone therapy to have MG. Here, the histomorphological and histochemical changes that were seen in muscle biopsies of MG with atypical clinical presentation were the followings: atrophy of type II fibers; predominance of type I fibers; increased NADH staining at the neuromuscular junction (NMJ). The most consistent finding is marked reduction in acetylcholinesterease (AChE) activity at the end plates in all cases. The results of this study suggest that histochemical and acetyl cholinesterase stains, in combination with clinical assessment can help to identify unsuspected cases of MG.
\end{abstract}

Volume 4 Issue 6 - 2017

\author{
Hussam Abu Farsakh, ' Altaf ljmail ${ }^{2}$ \\ 'Department of Pathology, University of Rochester, USA \\ 2Department of Pathology, First Medical Lab, Jordan \\ Correspondence: Hussam Abu Farsakh, Department of \\ Pathology, University of Rochester, Rochester, New York and \\ First Medical Lab Director, 5th circle, near Sheraton Hotel, \\ Building no. 14,Amman, Jordan, Tel+ 9626593 | 3 | |0, Fax + 962 \\ 6593 1315, Email FIlab@yahoo.com
}

Received: June 04, 2017 | Published: June 21, 2017

Keywords: muscle biopsies, myasthenia gravis, acetyl cholinesterase receptor

Abbreviations: NMJ, neuromuscular junction; MB, muscle biopsies; AChE, acetylcholinesterease; MG, myasthenia gravis; NADH-TR, tetrazolium reductase; SDH, succinic dehydrogenase; AChE, acetyl cholinesterase; AChRA, acetylcholine receptor antibody

\section{Introduction}

Myasthenia gravis (MG) is a relatively rare autoimmune disorder in which antibodies form against acetylcholine nicotinic postsynaptic receptors at the neuromuscular junction of skeletal muscles. ${ }^{1,2}$ The disease is characterized by variable weakness and fatigability of oculobulbar and limb muscles. ${ }^{3}$ It has a bimodal peak of incidence with first peak in the third decade and the second peak in the sixth decade. ${ }^{4}$ The diagnosis is usually made on clinical and electrophysiological grounds. In the majority of patients, initial presentation is due to the involvement of extra ocular muscles. With the progression of the disease, facial, bulbar, proximal limb muscles, neck extensors, and diaphragm get involved. ${ }^{5-7}$

This disease has a prevalence of $2 / 10000$ population. Untreated MG has a 10year mortality of $20-30 \%$ and $85 \%$ of the patients are seropositive for acetyl choline antibodies. ${ }^{8,9}$ The histomophology and histochemical description of MG in muscle biopsy is rare. This may be due to the fact that most of the patients are diagnosed by clinical examination, serology, EMG and nerve conduction studies. However, in some cases, the clinical picture may not be typical and serology tests may either be negative or not available. In this article, we are describing the muscle morphological and histochemical changes of MG. Knowing these changes are essential for neuropathogiest so as not to miss cases with atypical presentation. We would like also to emphasize the value of routine use of acetylcholinesterease (AChE) staining in muscle biopsy.

\section{Patients and methods}

We studied 6patients who visited The First medical Lab in Amman, Jordan, during a 5year period. Age ranged from 11 to 60years $(5 \mathrm{~F}$, $1 \mathrm{M})$.

\section{Clinical evaluation}

None of patients had an initial diagnosis of myasthenia gravis at the time of presentation. Data were collected regarding muscle power, pattern of weakness, functional abilities, EMG studies, acetylcholine receptor antibodies.

\section{Muscle biopsy}

The muscle biopsy was performed using 14gauge tru cut needle through a small knife opening of $2 \mathrm{~mm}$ in diameter in the skin overlying the right quadriceps muscle, under local anesthesia. More than 30 passes were performed on each patient to collect enough muscle tissue material. The material was wrapped in sponge dampened with saline. A block with frozen O.C.T compound is prepared on a metal chip. All muscle material were mounted on the metal chip and covered by the O.C.T compound. A metal beaker filled with isopentane was dipped in liquid nitrogen for container. The mounted biopsy is then immersed in the cooled isopentane for 15 seconds. The frozen biopsy was removed and rapidly placed in $-20 \mathrm{C}$ freezers. Later, the muscle was being cut by the cryostat by $5 \mu \mathrm{m}$. The cut tissue was stored at -20 freezers for final staining.

\section{Morphological investigations}

Diagnostic needle muscle specimens were obtained from the quadriceps or the deltoid muscles of all patients. Standard histological and histochemical analysis was performed for the specimens of all patients as described. ${ }^{10} 5 \mu \mathrm{m}$ serial cross-sections were cut in a cryostat. Sections were stained with hematoxylin-eosin, modified 
trichrome stain, NADH tetrazolium reeducate (NADH-TR), succinic dehydrogenises (SDH), myofibrillar ATPase at $\mathrm{pH}$ 10.6., 4.2, 4.6, and acetyl cholinesterase (AChE).

\section{Results}

\section{Clinical examination}

Table 1 show that we have two peaks of occurrences. One peak at the second decade: five patients (age 11-21years), and another peak at the sixth decade one patient (age 60).

\section{Histological analysis}

Routine histological analysis of the quadriceps or deltoid muscle specimen of all cases showed mild variation of fiber size with interspersed mildly atrophic fibers (Figure 1). The abnormal variability of fiber size with type I fibers predominance was clearly demonstrated in Figure 2 and were seen in cases no.1,4\&5. The histochemical examination of the muscle biopsies of all patients revealed marked reduction in ACHE activity (Figure 3). There was increased oxidative enzyme NADH reaction at the neuromuscular junction in all cases but case no.3 (Figure 4). Table 2 shows the characteristics of the histochemical changes in six myasthenia gravis patients. The histological pictures in all cases showed almost common features of the following: minimal muscle fiber variation in size even in cases with long standing myasthenia gravis, and showed occasional atrophied fibers. In three of the six patients, predominance of type I fibers were observed, the atrophied fibers tended to be of type II. The most consistent finding in all cases was marked reduction in AChE staining at the end plates, However, increased NADH at the end plate were seen in five of the six patients. None of the cases showed lymphorrhagia that had been described before. In three of the six patients, electron microscopy was performed. In two of three cases mild increase in glycogen was noted and in one case, glycogen increase was coupled with increased fat globules.

Acetylcholine receptor antibody (AChRA) was positive only in one case out of six cases $(16 \%)$. All patients responded dramatically to Acetylcholinesterease inhibitors (like mestinon) and thymectomy was performed in case no.2. Thymic hyperplasia was seen on microscopic examination.

Table I Clinical details and laboratory features of the six patients

\begin{tabular}{|c|c|c|c|c|c|c|}
\hline Patient & $I(m n 23.4 c)$ & $2(m n \mid 8.4 b)$ & $3(\mathrm{mn} \mid \mathrm{I} .4 \mathrm{c})$ & $4(\mathrm{mn} \mid 7.4 \mathrm{c})$ & $5(\mathrm{mn} 2 \mathrm{I} .4 \mathrm{c})$ & $6(\mathrm{mn} 2.5 \mathrm{~b})$ \\
\hline Age(yrs)/sex & $12 / F$ & $15 / F$ & $I I / M$ & $60 / F$ & $2 \mathrm{I} / \mathrm{F}$ & $20 / F$ \\
\hline $\begin{array}{l}\text { Duration of } \\
\text { symptoms before } \\
\text { diagnosis (yrs) }\end{array}$ & 11 & 2 & 10 & 6 & 5 & I \\
\hline Pattern of weakness & $\begin{array}{l}\text { proximal } \\
\text { muscles }\end{array}$ & $\begin{array}{l}\text { proximal } \\
\text { muscles }\end{array}$ & $\begin{array}{l}\text { Proximal } \\
\text { muscles }\end{array}$ & $\begin{array}{l}\text { extra ocular } \\
\text { muscles }\end{array}$ & neck muscles & $\begin{array}{l}\text { proximal } \\
\text { muscles }\end{array}$ \\
\hline Ptosis & absent & absent & Present & present & present & absent \\
\hline Diurnal variation & none & none & none & none & none & none \\
\hline CPK & normal & normal & Normal & normal & normal & mildly raised \\
\hline Family history & no & no & $\begin{array}{l}\text { Yes (one } \\
\text { brother) }\end{array}$ & no & no & no \\
\hline Consanguinity & no & no & Yes & no & no & no \\
\hline EMG & myopathic & myopathic & myopathic & myopathic & myopathic & myopathic \\
\hline AChR Antibody & negative & negative & Negative & negative & negative & negative \\
\hline Response to therapy & $\begin{array}{l}\text { Mestinon } \\
\text { improved }\end{array}$ & $\begin{array}{l}\text { Mestinon followed } \\
\text { by thymectomy } \\
\text { improved }\end{array}$ & $\begin{array}{l}\text { Mestinon } \\
\text { improved }\end{array}$ & $\begin{array}{l}\text { Mestinon } \\
\text { improved }\end{array}$ & $\begin{array}{l}\text { Mestinon } \\
\text { improved }\end{array}$ & $\begin{array}{l}\text { Mestinon } \\
\text { improved }\end{array}$ \\
\hline
\end{tabular}


Table 2 Summary of the morphological changes in MG patients

\begin{tabular}{|c|c|c|c|c|c|c|}
\hline Patient & $I(\mathrm{mn} 23.4 \mathrm{c})$ & $2(\mathrm{mn} \mid 8.4 \mathrm{~b})$ & $3(\mathrm{mnI} I .4 \mathrm{c})$ & $4(\mathrm{mn} \mid 7.4 \mathrm{c})$ & $5(\mathrm{mn} 2 \mathrm{I} .4 \mathrm{c})$ & $6(\mathrm{mn} 2.5 \mathrm{~b})$ \\
\hline Muscle biopsies & R. Quadriceps & L. deltoid & R. Quadriceps & R. Deltoid & R. Quadriceps & R. Quadriceps \\
\hline Atrophic fibers & type II & type II & type II & both types & type II & type II \\
\hline $\begin{array}{l}\text { NADH stain at } \\
\text { Neuromuscular junction }\end{array}$ & increase & increase & normal & increase & increase & increase \\
\hline Fiber type predominance & type I & type I and II & type I and II & type I & type I & type I and II \\
\hline Muscle necrosis & no & no & no & no & no & no \\
\hline Muscle inflammation & no & no & no & no & no & no \\
\hline Lymphorrahgia & no & no & no & no & no & no \\
\hline Centronucleation & $<5 \%$ & $<5 \%$ & $10 \%$ & $<5 \%$ & $<5 \%$ & $<5 \%$ \\
\hline Fibrosis & no increase & no increase & no increase & no increase & no increase & no increase \\
\hline $\begin{array}{l}\text { Acetyl cholinesterase at } \\
\text { neuromuscular junction }\end{array}$ & decrease & decrease & decrease & decrease & decrease & decrease \\
\hline
\end{tabular}

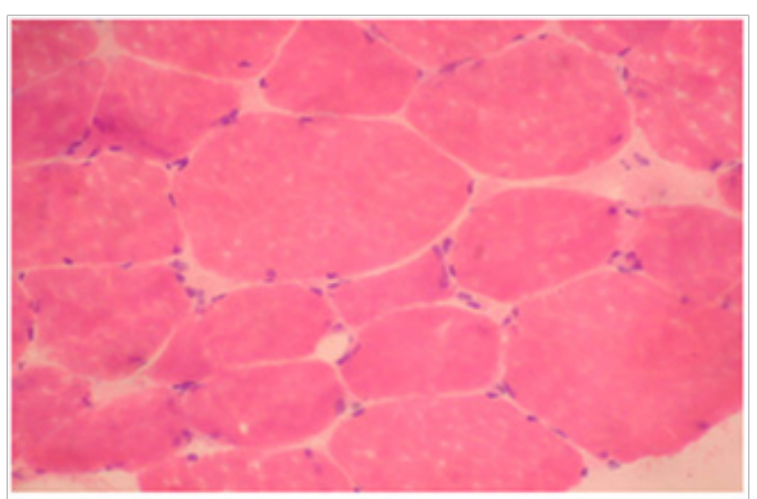

Figure I Quadriceps muscle biopsy of patient I. Hematoxyline and eosin sections showing variation in fiber size with interspersed mildly atrophic fibers. The atrophic fibers are seen selectively in type II fibers (Mag.400x).

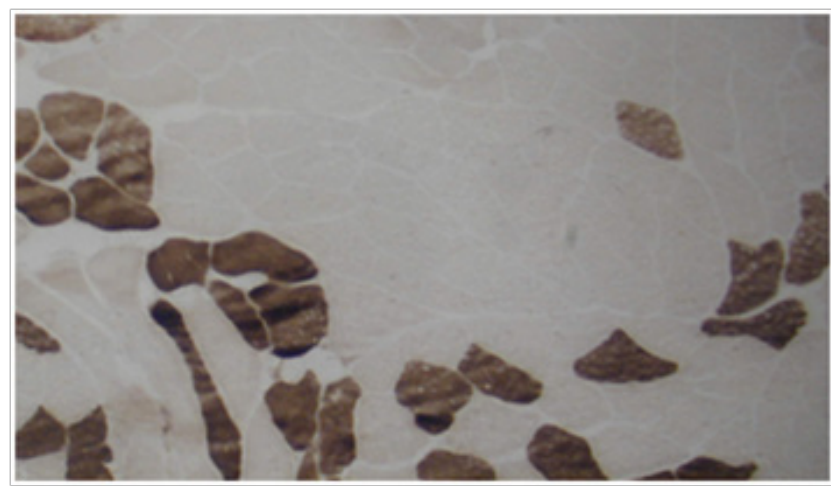

Figure 2 Quadriceps muscle biopsy of case I with alkaline preincubation ATPase stain at $\mathrm{pH} 10.6$ showing predominance of type I fibers (Mag.400).

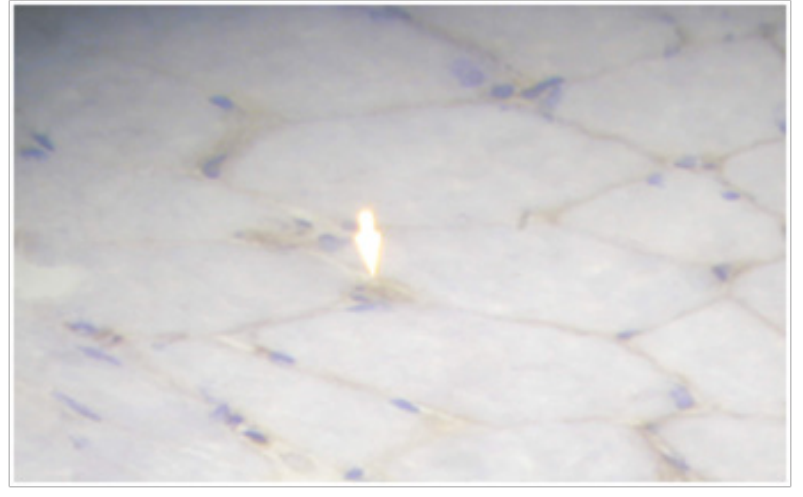

Figure 3 Quadriceps muscle biopsy of case 2 showing marked reduction of A chase activity (Mag.400x).

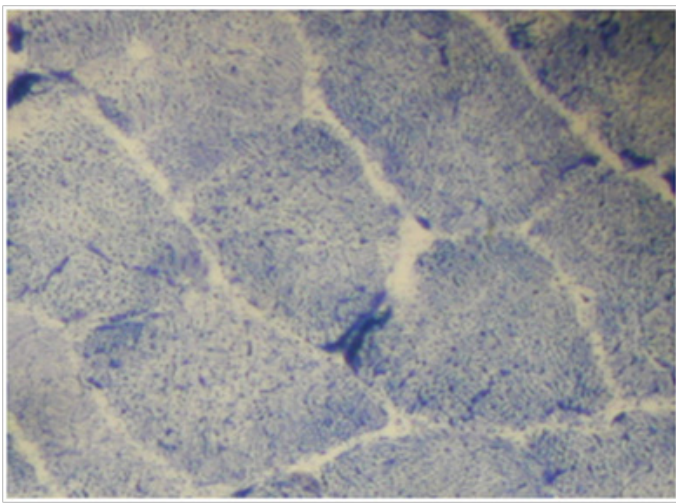

Figure 4 Quadriceps muscle biopsy of case 2 showing muscle fibers with increased NADH staining at the NMJ. 


\section{Discussion}

The six cases of myasthenia gravis reported in this study showed some features similar to those reported in the description by Dubowitz. ${ }^{9-11}$ Except for Dubowitz description, the recent literature is lacking significantly, any other descriptive features of histochemical staining for MG. In our study, the six patients constitute $3.3 \%$ of 182patients observed in a 5-year period. All patients represented a diagnostic challenge. For them, different diagnoses other than MG have been previously proposed, including myositis, motor neuron diseases, limb girdle muscular dystrophies, or mitochondrial myopathies. Understanding that MG can have atypical clinical presentation is very important for their diagnosis. While $90 \%$ of patient with MG are reported to have AChRA ${ }^{12}$ patients with atypical clinical presentation that come to muscle biopsy may have much less chance of having positive AChRA. In our series, only one out of six cases $(16 \%)$ had positive AChRA In that case, his clinician ordered the AChRA only after we made the diagnosis based on histochemical and AChE staining. The inability of recognizing the morphological changes of MG by the pathologist, render diagnosing such cases correctly almost impossible. In our series, the duration of patients symptoms ranged from 1 year to 11years. We strongly emphasize the use of AChE staining in any case that the pathologist sees minimal variation of muscle fibers disproportionate to the muscle power of the patient. The decreased AChE staining has been reported in Washington's University web site, ${ }^{13}$ and we agree with their report.

Thymic hyperplasia is noted in $75 \%$ of patients with MG. Of these, germinal hyperplasia is noted in $85 \%$ and thymic tumor in $15 \%{ }^{3}$ Thymectomy, a known modality for the treatment of MG, induced excellent response in case no.2. None of the other cases had thymectomy. None of our patients had typical diurnal variation seen in MG with better power in the morning. Most of the affected muscles were the facial muscle, neck muscle and proximal upper and lower limb muscles. This selective pattern of muscle involvement may hypothesize a selective functional alteration of neuromuscular transmission. ${ }^{14}$ The pathological mechanism leading to selective proximal muscle weakness and wasting in MG patients may be related to the presence of unknown auto antibodies or specific immunologic profile. ${ }^{14,15}$

It is interesting to report that lymphorrhagia which has been described by Russell, Adams et al., ${ }^{16}$ Engel \& McFarlin, ${ }^{17}$ is not seen in any of our 6cases and it is not a constant finding. ${ }^{16,17}$ Dubowitz has emphasized the presence of type II atrophy with patchy distribution in 4 out of 6cases of his study, he noticed type I fiber atrophy in some areas in 4 out of 6 cases. He suggested that the reason for these patchy changes were not all motor units myasthenia. ${ }^{9-11}$ The increased NADH staining at the NMJ has been described by Rodolico as increased tubular aggregates at the NMJ in patient with $\mathrm{MG} .{ }^{14}$ We observed this finding in 5 out of 6 sixcases, and helped us in making the diagnosis in these cases. However, this finding is not specific and has been observed in cases other than MG in our entire cases. In conclusion, MG is a common muscle disease, but it comes to muscle biopsy only rarely because most of these patients are diagnosed based on clinical and serology grounds only. Pathologists may miss the diagnosis of $\mathrm{MG}$ if he is not aware of the changes that occur in such cases or AChE staining is not available in his lab.

\section{Acknowledgements}

None.

\section{Conflict of interest}

The author declares no conflict of interest.

\section{References}

1. Strauss AJL, Seigal BC, Hsu KC. Immunofluorescence demonstration of a muscle binding complement fixing serum globulin fraction in Myasthenia gravis. Proc Soc Exp Biol. 1960;105:184.

2. Patric J, Lindstrom JM. Autoimmune response to acetylcholine receptor. Science. 1973;180(4088):871-872.

3. Oosterhuis HJ. The natural course of myasthenia gravis: a long term follow up study. J Neurol Neurosurg Psychiatry. 1989;52(10):1121-1127.

4. Drachman D. Myasthenia gravis. In: Rose N, et al. editors. The autoimmune diseases. 3rd ed. Massachusetts, USA: Academic Press; 1998. p. 637-662.

5. MacDonald BK, Cockerell OC, Sander JW, et al. The incidence and life time prevalence of neurological disorders in a prospective community based study. Brain. 2003;123(40):665-676.

6. Engel AG. Acquired autoimmune myasthenia gravis. In: Engel AG, et al. editors. Myology: Basic and Clinical. 2nd ed. New York, USA: Oxford University Press; 1999.

7. J P Sieb. Myasthenia gravis: an update for the clinician. Clin Exp Immunol. 2014;175(3):408-418

8. Hill M. The neuromuscular junction disorders. J Neurol Neurosurg Psychiatry. 2003;74(suppl 2):ii32-ii37.

9. Dubowitz V. Muscle Biopsy: a practical approach. 2nd ed. Bailliere Tindall, London, UK: Academic Press; 1985.

10. Dubowitz V. Muscle disorders in Childhood. London: WB Saunders. 1978; $16: 282$.

11. Dubowitz V. $50^{\text {th }}$ ENMC International workshop: congenital muscular dystrophy, 28 February 1997 to 2 March 1997. Naarden, the Netherlands. Neuromuscul Disord. 1990;7(8):539-547.

12. Vincent A, Clover L, Buckley C, et al. Myasthenia gravis survey evidence of under diagnosis of myasthenia gravis in older people. $J$ Neurol Neurosurg Psychiatry. 2003;74(8):1105-1108.

13. Myasthenia gravis \& neuromuscular junction (NMJ) disorders.

14. Aragones JM, Bolibar I, Bonfill X, et al. Myasthenia gravis: a higher than expected incidence in the elderly. Neurology. 2003;60(6):1024-1026.

15. Rodolico C, Toscano A, Autunno M, et al. Limb-girdle myasthenia: clinical, electrophysiological and morphological features in familial and autoimmune cases. Neuromuscul Disord. 2002;12(10):964-969.

16. Adams RD, Denny-Brown D, Pearson CM. Diseases of Muscle. A Study in pathology. 1st ed. New York, USA: Hoeber; 1953.

17. Engel WK, McFarlui DE. Discussion of paper by Fenichel. USA: Ann NY Acad Set; 1966. p. 135-168. 